\title{
Fast and isothermal hydrothermal liquefaction of polysaccharide feedstocks
}

\section{Akhila Gollakota $^{1}$ and Phillip E. Savage ${ }^{1 *}$}

${ }^{1}$ Department of Chemical Engineering, 121D Chemical and Biomedical Engineering Building, The Pennsylvania State University, University Park, PA 16802, United States

* Corresponding author

E-mail: psavage@psu.edu

\section{Pages}

1 Table 
Table S1 Tentative identities of organic compounds in biocrudes produced by isothermal HTL at $350{ }^{\circ} \mathrm{C}$ set-point temperature, 31.6 min and fast HTL at $500^{\circ} \mathrm{C}$ set-point temperature, 1 min for the polysaccharide feedstocks

\begin{tabular}{|c|c|c|c|c|c|c|c|c|c|c|c|c|c|}
\hline \multirow{2}{*}{$\begin{array}{l}\text { Compound } \\
\text { Group }\end{array}$} & \multirow[t]{2}{*}{ Compound Identified } & \multicolumn{2}{|c|}{ Starch } & \multicolumn{2}{|c|}{ Cellulose } & \multicolumn{2}{|c|}{ Amylopectin } & \multicolumn{2}{|c|}{ Amylose } & \multicolumn{2}{|c|}{ Pectin } & \multicolumn{2}{|c|}{ Chitin } \\
\hline & & Iso. & Fast & Iso. & Fast & Iso. & Fast & Iso. & Fast & Iso. & Fast & Iso. & Fast \\
\hline \multirow{35}{*}{ Ketones } & 2-Cyclopenten-1-one,3-methyl- & $\checkmark$ & & $\checkmark$ & & $\checkmark$ & & $\checkmark$ & & $\checkmark$ & & $\mathrm{c}$ & \\
\hline & 2-Cyclopenten-1-one,2,3-dimethyl- & $\checkmark$ & & $\checkmark$ & & $\checkmark$ & & $\checkmark$ & & $\checkmark$ & $\checkmark$ & $\checkmark$ & \\
\hline & 2-Cyclopenten-1-one & $\checkmark$ & & $\checkmark$ & & $\checkmark$ & $\checkmark$ & $\checkmark$ & & $\checkmark$ & & & \\
\hline & Ethanone,1-(3-hydroxyphenyl)- & $\checkmark$ & $\checkmark$ & $\checkmark$ & & $\checkmark$ & & & $\checkmark$ & & $\checkmark$ & & \\
\hline & 3,6-Heptanedione & & & $\checkmark$ & & $\checkmark$ & $\checkmark$ & $\checkmark$ & & & & & \\
\hline & $\begin{array}{l}\text { 2-Cyclohexen-1-one, 6-methyl-3-(1- } \\
\text { methylethyl)- }\end{array}$ & $\checkmark$ & & $\checkmark$ & & $\checkmark$ & & & & & & $\checkmark$ & \\
\hline & 2,5-Hexanedione & $\checkmark$ & & $\checkmark$ & & $\checkmark$ & $\checkmark$ & $\checkmark$ & & & & & \\
\hline & 2-Cyclopenten-1-one,2-methyl- & $\checkmark$ & & $\checkmark$ & & $\checkmark$ & & $\checkmark$ & & $\checkmark$ & & $\checkmark$ & \\
\hline & 1,2-Cyclopentanedione,3-methyl- & $\checkmark$ & & $\checkmark$ & & & & $\checkmark$ & & & & & \\
\hline & 2-Cyclopenten-1-one,3-ethyl- & $\checkmark$ & & $\checkmark$ & & $\checkmark$ & & $\checkmark$ & & $\checkmark$ & & $\checkmark$ & \\
\hline & Ethanone,1-(1-cyclohexen-1-yl)- & $\checkmark$ & & $\checkmark$ & & & & $\checkmark$ & & $\checkmark$ & & $\checkmark$ & \\
\hline & Cyclohexanone,3-ethenyl- & $\checkmark$ & & $\checkmark$ & & $\checkmark$ & & $\checkmark$ & & $\checkmark$ & & $\checkmark$ & \\
\hline & 2-Cyclopenten-1-one,3,4-dimethyl- & $\checkmark$ & & $\checkmark$ & & $\checkmark$ & & $\checkmark$ & & $\checkmark$ & & $\checkmark$ & \\
\hline & 4,4-Dimethyl-2-cyclopenten-1-one & $\checkmark$ & & $\checkmark$ & & & & $\checkmark$ & & $\checkmark$ & & $\checkmark$ & \\
\hline & 2-Acetonylcyclopentanone & $\checkmark$ & & $\checkmark$ & & & & & & & & & \\
\hline & $\begin{array}{l}\text { 1,3-Cyclopentanedione, } 2,4- \\
\text { dimethyl- }\end{array}$ & $\checkmark$ & & & & & & & & & & & \\
\hline & Cyclohept-4-enone & $\checkmark$ & & & & & & & & & & & \\
\hline & $\begin{array}{l}\text { 2-Cyclopenten-1-one, 3-ethyl-2- } \\
\text { hydroxy- }\end{array}$ & $\checkmark$ & $\checkmark$ & & & $\checkmark$ & & & & & & & \\
\hline & 3-Methylcyclopentane-1,2-dione & & & & & $\checkmark$ & & & & & & & \\
\hline & 3,4-dihydroxyphenyl-2-propanone & & & & & $\checkmark$ & & & & & & & \\
\hline & $\begin{array}{l}\text { 2-Cyclohexen-1-one, } \quad 3,4- \\
\text { dimethyl- }\end{array}$ & & & & & & & $\checkmark$ & & $\checkmark$ & & & \\
\hline & $\begin{array}{l}\text { 5-Hexen-2-one, 5-methyl-3- } \\
\text { methylene- }\end{array}$ & & & & & & & $\checkmark$ & & & & & \\
\hline & Cyclopentanone & & & & & & & & & $\checkmark$ & & $\checkmark$ & \\
\hline & 3-Methyl-3-cyclohexen-1-one & & & & & & & & & $\checkmark$ & & & \\
\hline & 2-Cyclohexen-1-one,3-methyl- & & & & & & & & & $\checkmark$ & & & \\
\hline & Cyclohexanone & & & & & & & & & $\checkmark$ & & & \\
\hline & $\begin{array}{c}\text { 2-Cyclopenten-1-one, } 2,3,4,5- \\
\text { tetramethyl- }\end{array}$ & & & & & & & & & & & $\checkmark$ & \\
\hline & 3-Methylcyclopentane-1,2-dione & & $\checkmark$ & & & & & & $\checkmark$ & & $\checkmark$ & & \\
\hline & Maltol & & $\checkmark$ & & & & & & $\checkmark$ & & & & \\
\hline & Ethanone,1-(2,5-dihydroxyphenyl)- & & $\checkmark$ & & & & & & & & $\checkmark$ & & \\
\hline & 1,2-Cyclopentanedione & & $\checkmark$ & & & & $\checkmark$ & & $\checkmark$ & & & & $\checkmark$ \\
\hline & $\begin{array}{l}\text { 2-Cyclohexen-1-one,4-(1- } \\
\text { methylethyl)- }\end{array}$ & & & & & & $\checkmark$ & & $\checkmark$ & & & & \\
\hline & $\begin{array}{l}\text { 5,9-Dodecadien-2-one,6,10- } \\
\text { dimethyl-,(E,E))- }\end{array}$ & & & & & & & & $\checkmark$ & & & & \\
\hline & $\begin{array}{l}\text { 3-Buten-2-one, } \\
\text { hydroxy-3-methoxyphenyl)- }\end{array}$ & & & & & & & & & & $\checkmark$ & & \\
\hline & $\begin{array}{l}\text { 2-Hydroxy-3-propyl-2-cyclopenten- } \\
\text { 1-one }\end{array}$ & & & & & & & & & & $\checkmark$ & & \\
\hline
\end{tabular}




\begin{tabular}{|c|c|c|c|c|c|c|c|c|c|c|c|c|c|}
\hline \multirow{2}{*}{$\begin{array}{l}\text { Compound } \\
\text { Group }\end{array}$} & \multirow[t]{2}{*}{ Compound Identified } & \multicolumn{2}{|c|}{ Starch } & \multicolumn{2}{|c|}{ Cellulose } & \multicolumn{2}{|c|}{ Amylopectin } & \multicolumn{2}{|c|}{ Amylose } & \multicolumn{2}{|c|}{ Pectin } & \multicolumn{2}{|c|}{ Chitin } \\
\hline & & Iso. & Fast & Iso. & Fast & Iso. & Fast & Iso. & Fast & Iso. & Fast & Iso. & Fast \\
\hline \multirow{10}{*}{ Phenols } & Phenol & $\checkmark$ & & $\checkmark$ & $\checkmark$ & $\checkmark$ & $\checkmark$ & $\checkmark$ & $\checkmark$ & $\checkmark$ & & $\checkmark$ & $\checkmark$ \\
\hline & Phenol,2-methyl- & $\checkmark$ & & $\checkmark$ & & $\checkmark$ & & $\checkmark$ & & $\checkmark$ & & $\checkmark$ & \\
\hline & Phenol,3-methyl- & $\checkmark$ & & $\checkmark$ & & $\checkmark$ & & $\checkmark$ & & $\checkmark$ & $\checkmark$ & $\checkmark$ & \\
\hline & Phenol,2,3-dimethyl- & & & $\checkmark$ & & & & & & & & & \\
\hline & Phenol,2,5-dimethyl- & & & & & & & & & $\checkmark$ & & & \\
\hline & Phenol,4-ethyl-2-methoxy- & & & & & & & & & $\checkmark$ & & & \\
\hline & Phenol,3,4-dimethyl- & & & $\checkmark$ & & & & $\checkmark$ & & & & & \\
\hline & $\begin{array}{c}\text { Phenol,2,2'-methylenebis[6-(1,1- } \\
\text { dimethylethyl)-4-methyl- }\end{array}$ & & $\checkmark$ & $\checkmark$ & $\checkmark$ & $\checkmark$ & & & & & $\checkmark$ & & \\
\hline & Phenol, 2-methoxy- & & & & & & & & & & & $\checkmark$ & \\
\hline & 2-Methoxy-5-methylphenol & & & & $\checkmark$ & & & & & & & & \\
\hline \multirow{6}{*}{$\begin{array}{l}\text { Indanone } \\
\text { derivatives }\end{array}$} & 1H-Inden-1-one,2,3-dihydro- & $\checkmark$ & & $\checkmark$ & & $\checkmark$ & & $\checkmark$ & & $\checkmark$ & & $\checkmark$ & \\
\hline & 7-Methylindan-1-one & $\checkmark$ & & $\checkmark$ & & $\checkmark$ & & $\checkmark$ & & $\checkmark$ & & & \\
\hline & Indan-1,2-dione, 4,7-dimethyl- & & & & & $\checkmark$ & & & & & & & \\
\hline & 1H-Inden-5-ol, 2,3-dihydro- & & & $\checkmark$ & & $\checkmark$ & & $\checkmark$ & & $\checkmark$ & & & \\
\hline & 4-Hydroxy-1-indanone & & & & & & & $\checkmark$ & & $\checkmark$ & & & \\
\hline & 1-Methylindan-2-one & & & & & & & & & $\checkmark$ & & & \\
\hline \multirow{18}{*}{$\begin{array}{c}\text { Furan } \\
\text { derivatives }\end{array}$} & 2-Methyl-5-hydroxybenzofuran & $\checkmark$ & $\checkmark$ & $\checkmark$ & & $\checkmark$ & & $\checkmark$ & & & $\checkmark$ & & \\
\hline & 2,4-Dimethylfuran & $\checkmark$ & $\checkmark$ & $\checkmark$ & & $\checkmark$ & & $\checkmark$ & & & & & \\
\hline & $2(3 \mathrm{H})$-Benzofuranone & & & & & $\checkmark$ & & & & & & & \\
\hline & Benzofuran,4,7-dimethyl- & & & & & & & $\checkmark$ & & & & & \\
\hline & Benzofuran,2-methyl- & & & & & & & $\checkmark$ & & $\checkmark$ & & & \\
\hline & 2-Furancarboxaldehyde, 5-methyl- & $\checkmark$ & & & $\checkmark$ & $\checkmark$ & $\checkmark$ & $\checkmark$ & $\checkmark$ & & & & $\checkmark$ \\
\hline & 5-Hydroxymethylfurfural & & $\checkmark$ & $\checkmark$ & $\checkmark$ & & $\checkmark$ & & $\checkmark$ & & $\checkmark$ & & $\checkmark$ \\
\hline & $\begin{array}{l}\text { Benzofuran-2,3-dione,4,7-dimethyl- } \\
\text { 2,3-dihydro- }\end{array}$ & $\checkmark$ & & & & & & & & & & & \\
\hline & $\begin{array}{c}\text { 2-Furancarboxylic acid,2- } \\
\text { methylpropyl ester }\end{array}$ & & $\checkmark$ & & & & & & & & & & \\
\hline & 2-Methyl-5-hydroxybenzofuran & & & & & & & & & & & & \\
\hline & 2,5-Furandicarboxaldehyde & & $\checkmark$ & & $\checkmark$ & & $\checkmark$ & & $\checkmark$ & & & & $\checkmark$ \\
\hline & Furyl hydroxymethyl ketone & & & & $\checkmark$ & & $\checkmark$ & & $\checkmark$ & & & & \\
\hline & Furfural & & & & & & $\checkmark$ & & & & & & \\
\hline & 2-Acetyl-5-methylfuran & & & & & & $\checkmark$ & & & & & & \\
\hline & 2(5H)-Furanone & & & & & & $\checkmark$ & & & & & & \\
\hline & 2,2'-Bifuran & & & & $\checkmark$ & & & & & & & & \\
\hline & 2(5H)-Furanone, 5,5-dimethyl- & & & & & & & & & & & & $\checkmark$ \\
\hline & 5-Acetoxymethyl-2-furaldehyde & & & & & & & & $\checkmark$ & & & & $\checkmark$ \\
\hline
\end{tabular}




\begin{tabular}{|c|c|c|c|c|c|c|c|c|c|c|c|c|c|}
\hline \multirow{2}{*}{$\begin{array}{l}\text { Compound } \\
\text { Group }\end{array}$} & \multirow[t]{2}{*}{ Compound Identified } & \multicolumn{2}{|c|}{ Starch } & \multicolumn{2}{|c|}{ Cellulose } & \multicolumn{2}{|c|}{ Amylopectin } & \multicolumn{2}{|c|}{ Amylose } & \multicolumn{2}{|c|}{ Pectin } & \multicolumn{2}{|c|}{ Chitin } \\
\hline & & Iso. & Fast & Iso. & Fast & Iso. & Fast & Iso. & Fast & Iso. & Fast & Iso. & Fast \\
\hline \multirow{18}{*}{$\begin{array}{c}\text { Benzene } \\
\text { derivatives }\end{array}$} & Catechol & & $\checkmark$ & & & $\checkmark$ & & & & $\sqrt{ }$ & $\checkmark$ & & \\
\hline & 1,2-Benzenediol,4-methyl- & $\checkmark$ & $\checkmark$ & $\checkmark$ & & $\checkmark$ & & & & $\checkmark$ & $\checkmark$ & & \\
\hline & 1,2-Benzenediol,3-methyl- & $\checkmark$ & $\checkmark$ & $\checkmark$ & & $\checkmark$ & & & & & $\checkmark$ & & \\
\hline & 2-Coumaranone & & & $\checkmark$ & & & & & & & & & \\
\hline & 1,3-Benzenediol,4-ethyl- & & $\checkmark$ & $\checkmark$ & & & & & & $\checkmark$ & & & \\
\hline & 1,4-Benzenediol,2-methyl- & & & & & & & & $\sqrt{ }$ & & & & \\
\hline & $\begin{array}{c}\text { 1,4-Benzenedicarboxaldehyde,2- } \\
\text { methyl- }\end{array}$ & $\checkmark$ & & & & & & & & $\checkmark$ & & & \\
\hline & Benzene,ethoxy- & & & & & & & & & & & $\checkmark$ & \\
\hline & Resorcinol,2-acetyl- & & $\checkmark$ & & & & & & & & & & \\
\hline & Benzaldehyde, 3-hydroxy- & & $\checkmark$ & & & & & & & & & & \\
\hline & Benzene,1,3-bis(1,1-dimethylethyl)- & & & & $\checkmark$ & & & & & & & & \\
\hline & $\begin{array}{c}\text { Benzaldehyde, 3-hydroxy-3- } \\
\text { Formylphenol }\end{array}$ & & & & & & & & & & $\checkmark$ & & \\
\hline & Resorcinol,2-acetyl- & & & & & & & & & & $\checkmark$ & & \\
\hline & Hydrocoumarin & & & & & & & & & & $\checkmark$ & & \\
\hline & 4-Hydroxy-2-methylbenzaldehyde & & & & & & & & & & $\checkmark$ & & \\
\hline & 3-Hydroxy-2-methylbenzaldehyde & & & & & & & & & & $\checkmark$ & & \\
\hline & Vanillin & & & & & & & & & & $\checkmark$ & & \\
\hline & 4-Ethoxystyrene & & & & & & & & & & $\checkmark$ & & \\
\hline \multirow[t]{2}{*}{ Acids } & $\begin{array}{c}\text { 2-Hexenoic acid,3,4,4-trimethyl-5- } \\
\text { oxo-,(Z)- }\end{array}$ & & $\checkmark$ & & & & & & & & & & \\
\hline & n-Hexadecanoic acid & & & & $\checkmark$ & & $\checkmark$ & & $\sqrt{ }$ & & & & \\
\hline \multirow[t]{5}{*}{ Alcohols } & 1-Dodecanol & & & & $\checkmark$ & & $\checkmark$ & & $\checkmark$ & & & & \\
\hline & 1-Decanol,2-hexyl- & & & & $\checkmark$ & & & & $\sqrt{ }$ & & & & \\
\hline & 1-Decanol, 2-octyl- & & & & $\checkmark$ & & & & & & & & \\
\hline & n-Nonadecanol-1 & & & & $\checkmark$ & & & & & & & & \\
\hline & 2-Isopropyl-5-methyl-1-heptanol & & & & & & & & $\checkmark$ & & & & \\
\hline \multirow[t]{2}{*}{ Esters } & 2-Propenoic acid, pentadecyl ester & & & & $\checkmark$ & & $\checkmark$ & & $\checkmark$ & & & & \\
\hline & $\begin{array}{l}\text { Hexadecanoic acid,2-hydroxy-1- } \\
\text { (hydroxymethyl) ethyl ester }\end{array}$ & & & & $\checkmark$ & & $\checkmark$ & & & & & & \\
\hline \multirow[t]{6}{*}{ Hydrocarbons } & Nonane,5-butyl- & & & & & & $\checkmark$ & & & & & & \\
\hline & Eicosane & & & & $\checkmark$ & & & & $\checkmark$ & & & & \\
\hline & Heptadecane & & & & $\checkmark$ & & & & $\checkmark$ & & & & $\checkmark$ \\
\hline & Tetracosane & & & & & & & & & & & & $\checkmark$ \\
\hline & Tetratriacontane & & & & & & & & & & & & $\checkmark$ \\
\hline & Hexatriacontane & & & & & & & & & & & & $\checkmark$ \\
\hline
\end{tabular}




\begin{tabular}{|c|c|c|c|c|c|c|c|c|c|c|c|c|c|}
\hline \multirow{2}{*}{$\begin{array}{l}\text { Compound } \\
\text { Group }\end{array}$} & \multirow[t]{2}{*}{ Compound Identified } & \multicolumn{2}{|c|}{ Starch } & \multicolumn{2}{|c|}{ Cellulose } & \multicolumn{2}{|c|}{ Amylopectin } & \multicolumn{2}{|c|}{ Amylose } & \multicolumn{2}{|c|}{ Pectin } & \multicolumn{2}{|c|}{ Chitin } \\
\hline & & Iso. & Fast & Iso. & Fast & Iso. & Fast & Iso. & Fast & Iso. & Fast & Iso. & Fast \\
\hline \multirow{20}{*}{$\begin{array}{l}\text { Nitrogenous } \\
\text { compounds }\end{array}$} & 2,5-Pyrrolidinedione, 1-methyl & & & & & & & & & & & $\checkmark$ & \\
\hline & 2-acetyl pyrrole & & & & & & & & & & & $\checkmark$ & $\checkmark$ \\
\hline & Pyridine & & & & & & & & & & & $\checkmark$ & \\
\hline & 1H-Indole, 2,3-dimethyl- & & & & & & & & & & & $\checkmark$ & \\
\hline & Pyridine, 4-propyl- & & & & & & & & & & & $\checkmark$ & \\
\hline & Pyrazine, methyl- & & & & & & & & & & & $\checkmark$ & \\
\hline & 2,6-Dimethylphenyl isocyanate & & & & & & & & & & & $\checkmark$ & \\
\hline & Pyrazine, 2-ethyl-3-methyl- & & & & & & & & & & & $\checkmark$ & \\
\hline & 2-Acetyl-1-methylpyrrole & & & & & & & & & & & $\checkmark$ & \\
\hline & 2,5-Pyrrolidinedione, 1-ethyl & & & & & & & & & & & $\checkmark$ & \\
\hline & Pyridine, 2-methyl- & & & & & & & & & & & $\checkmark$ & \\
\hline & 1,3-Benzoxazol-4-ol & & & & & & & & & & & $\checkmark$ & \\
\hline & Acetamide & & & & & & & & & & & $\checkmark$ & \\
\hline & 1H-Pyrrole-2-carboxaldehyde & & & & & & & & & & & & $\checkmark$ \\
\hline & $\begin{array}{l}\text { 1H-Pyrrole-2-carboxaldehyde, 1- } \\
\text { methyl- }\end{array}$ & & & & & & & & & & & & $\sqrt{ }$ \\
\hline & 2-Acetoxymethylpyridine & & & & & & & & & & & & $\checkmark$ \\
\hline & $\begin{array}{l}\text { 4(1H)-Pyridinone, 2,3-dihydro-1- } \\
\text { methyl- }\end{array}$ & & & & & & & & & & & & $\checkmark$ \\
\hline & $\begin{array}{l}\text { 3-(Furan-2-yl)-4-methyl-1,2,4- } \\
\text { triazole }\end{array}$ & & & & & & & & & & & & $\checkmark$ \\
\hline & $\begin{array}{l}\text { Acetamide, N-(2,4- } \\
\text { dihydroxyphenyl)- }\end{array}$ & & & & & & & & & & & & $\checkmark$ \\
\hline & $\begin{array}{c}\text { 4-Hydroxy-6- } \\
\text { (methylamino)pyrimidine }\end{array}$ & & & & & & & & & & & & $\checkmark$ \\
\hline
\end{tabular}

